\title{
Concurrent substitutional and displacive phase transformations in Al-Mg-Si nanoclusters
}

\author{
M. A. van Huis, ${ }^{1,2, *}$ M. H. F. Sluiter, ${ }^{2,3}$ J. H. Chen, ${ }^{1,2}$ and H. W. Zandbergen ${ }^{1}$ \\ ${ }^{1}$ National Centre for HREM, Kavli Institute of Nanoscience, Delft University of Technology, Lorentzweg 1, NL-2628 CJ Delft, \\ The Netherlands \\ ${ }^{2}$ Netherlands Institute for Metals Research, Delft University of Technology, Mekelweg 2, NL-2628 CD Delft, The Netherlands \\ ${ }^{3}$ Laboratory of Materials Science, Delft University of Technology, Mekelweg 2, NL-2628 CD Delft, \\ The Netherlands
}

(Received 24 September 2007; published 26 November 2007)

\begin{abstract}
Al-Mg-Si nanoclusters embedded in aluminum transform through a delicate interplay of two characteristically distinct phase transformations: a diffusion-controlled substitutional transformation associated with solute enrichment and a swift displacive transformation involving collective shifts of columns of atoms once a critical enrichment has been reached. Through first-principles calculations, we show that these transformations are inseparable. Moreover, we show in atomistic detail how precipitates that are not superstructures of the matrix form from a supersaturated solid solution.
\end{abstract}

DOI: $10.1103 /$ PhysRevB.76.174113

PACS number(s): 64.70.Nd, 61.46.Df, 64.60.-i, 68.65.-k

\section{INTRODUCTION}

In many engineering alloys, precipitation is used to obtain optimal strength and ductility. Precipitates are small, secondphase nanoclusters that form and grow from an oversaturated solid solution, the so-called matrix, during a heat treatment process. It was discovered serendipitously already more than a century ago by Wilm ${ }^{1}$ in aluminum alloys and by the late 1930s the x-ray diffraction work of Guinier ${ }^{2}$ and Preston ${ }^{3}$ had shown that precipitation involved the formation of small nanoclusters. At present it is still unclear how the early stages of precipitation occur, especially when the precipitates take structures that have no obvious relation to the structure of the solid solution, usually referred to as a matrix. Al$\mathrm{Mg}-\mathrm{Si}$ alloys are a typical example: they have important applications in the automotive industry and the precipitation process is here of critical importance for obtaining the required mechanical properties, yet many of the precipitate structures are not superstructures of the fcc matrix and the early stage precipitates undergo a series of structural and compositional changes, leading to the formation of the $\beta^{\prime \prime}$ $\mathrm{Mg}_{5} \mathrm{Si}_{6}$ phase. ${ }^{4-6}$ Figure 1 shows an early needle-shaped $\mathrm{Mg}_{x} \mathrm{Si}_{y} \mathrm{Al}_{z}$ nanocluster as embedded in aluminum. The typical dimensions of these needles are $4 \times 4 \times 50 \mathrm{~nm}^{3}$, or even smaller. During heat treatment of $\mathrm{Al}-\mathrm{Mg}-\mathrm{Si}$ alloys the needles transform from the pre- $\beta^{\prime \prime}$ to the $\beta^{\prime \prime}$ phase, which is accompanied by a large increase in strength. Changes in these nanoclusters can be classified as solute enrichment, (partial) order-disorder transformations, and displacive transformations. ${ }^{4-7}$

So far, these transformations are generally considered independent. Here, we show that this is not the case. We show by means of first-principles calculations that in $\mathrm{Al}-\mathrm{Mg}-\mathrm{Si}$ alloys the nanocluster evolution can be described only by an entangled combination of phase transformations: a slowly progressing substitutional order-disorder transformation leads to changes in composition, whereby atomic relaxations enable a nonsubstitutional, displacive transformation along a single atomic column.

\section{THEORETICAL AND EXPERIMENTAL BACKGROUND}

A. Substitutional and displacive transformations

In dilute alloys segregation of alloying elements can occur gradually through spinodal decomposition ${ }^{8,9}$ and is some-

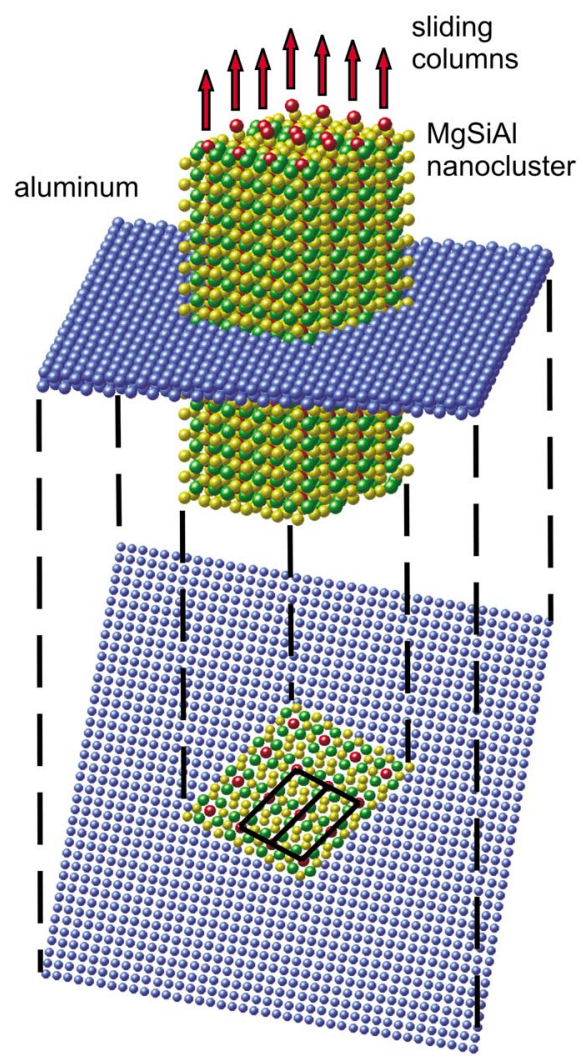

FIG. 1. (Color online) Illustration of a needle-shaped $\mathrm{Mg}_{4} \mathrm{Si}_{6} \mathrm{Al}$ nanocluster as embedded in aluminum (blue). Only two Al planes are shown for clarity. The needle consists of $\mathrm{Mg}$ atoms (green), $\mathrm{Si}$ atoms (yellow), and $\mathrm{Al}$ atoms. The columns of shifting $\mathrm{Al}$ atoms (red) within the nanocluster are indicated with arrows. At the bottom, two monoclinic unit cells are indicated. The crystal structure is shown in detail in Fig. 3. 
times followed by an ordering transformation once locally the composition is sufficiently enriched in alloying elements. Alternatively, segregation can occur abruptly through a classical nucleation and growth process of a new phase. ${ }^{10,11}$ Most gradual processes and many nucleation and growth processes are substitutional transformations whereby the atomic occupancy of sites in the crystal changes without obfuscating the parent crystal lattice. This can occur over extended length scales, such as in spinodal decomposition, or over short, interatomic length scales, such as in order-disorder phenomena. In either case the compositional fluctuations bring about changes in the periodicity without drastically affecting the lattice positions. When a nanocluster forms via such a substitutional mechanism it is then by definition a superstructure of the matrix. Atomic diffusion always plays a key role in the kinetics of these processes while the thermodynamic aspects of the formation of superstructures can be comprehensively explored through sophisticated computational tools, such as cluster expansions. ${ }^{12-15}$

A contrasting type of transformation is that of the displacive type. Here atomic diffusion is not required and no ordering transformations occur, but instead the crystal structure distorts homogeneously through collective shifts of atomic positions. This also leads to new crystal structures. Generally, displacive transformations are by their spatially global nature not associated with the highly localized phenomenon of precipitation. The kinetics of displacive transformations is generally rate limited. In a rate-limited transformation the transformation rate scales exponentially with activation energy (defined in Fig. 2) and inverse temperature, and linearly with time. In the case of displacive transformations, in general the activation energy is negligibly small [Fig. 2(b) evolves into Fig. 2(c)], which is why these transformations often propagate with velocities close to the velocity of sound waves in crystals, ${ }^{7}$ at a few hundred meters per second. In contrast, diffusion-limited phase transformations are much slower with rates scaling exponentially with the diffusion energy over the temperature and linearly with the square root of time. ${ }^{7}$

\section{B. Precipitation in Al-Mg-Si alloys}

The evolution of precipitation in Al-Mg-Si alloys has been extensively investigated experimentally. ${ }^{4-6,16,17}$ During the initial stages of precipitation, the atoms in the $\mathrm{Mg}_{x} \mathrm{Si}_{y} \mathrm{Al}_{z}$ nanoclusters share the same lattice positions as the $\mathrm{Al}$ host, the face-centered-cubic (fcc) matrix. At first, the solute $\mathrm{Mg}$ and $\mathrm{Si}$ atoms agglomerate into dilute clusters. ${ }^{16}$ Enrichment with solute atoms and growth follow, whereby more and more $\mathrm{Al}$ atoms in the nanocluster are replaced with $\mathrm{Mg}$ and $\mathrm{Si}$ atoms and whereby the nanoclusters grow from spheres into needles. ${ }^{4-6,16-18}$ The first evidence of ordering is the skeleton of $\mathrm{Si}-\mathrm{Si}$ columns that is formed in the initial $\beta^{\prime \prime}$ phase $^{6}$ and occurs approximately at the composition $\mathrm{Mg}_{2} \mathrm{Si}_{2} \mathrm{Al}_{7}$. Such $\mathrm{Si}_{2}$ columns are indicated in Fig. 3(c). The formation of the $\mathrm{Si}_{2}$ columns is a partial order-disorder transformation, as the lattice sites around the stable $\mathrm{Si}_{2}$ columns show a large variation of composition during the initial stages. Upon further maturing, more $\mathrm{Al}$ atoms are replaced a)

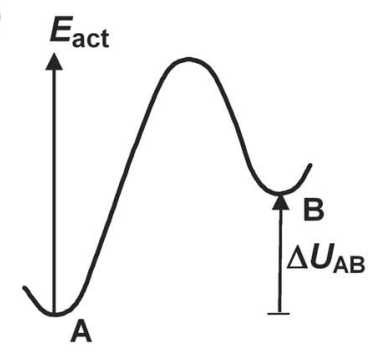

b)

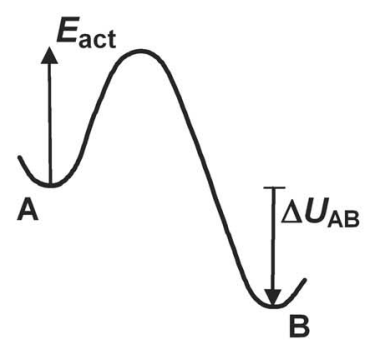

c)

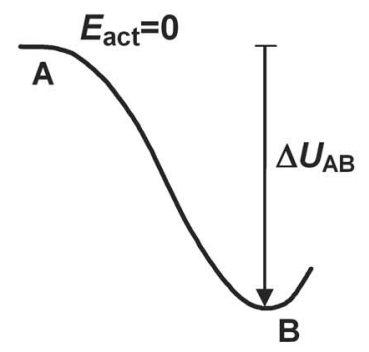

FIG. 2. Potential energy change during the transition from phase $A$ to phase $B$, with the activation energy $E_{\text {act }}$ and the energy difference $\Delta U_{A \rightarrow B}=U_{B}-U_{A}$. (a) Unfavorable transition $(\Delta U>0)$ with activation energy $E_{\text {act. }}$ (b) Favorable transition $(\Delta U<0)$ with activation energy $E_{\text {act }}$. (c) Favorable $(\Delta U<0)$, barrierless transition $\left(E_{\text {act }}=0\right)$. The energy profile of subfigure (b) is characteristic of a nucleation and growth process, while subfigure (c) is characteristic of a displacive transformation.

with $\mathrm{Mg}$ and $\mathrm{Si}$ atoms, gradually leading to the formation of the pre- $\beta^{\prime \prime}$ phase. Although these structures display variations in composition and have limited spatial dimensions, we speak about new phases as the symmetry operations change (space groups $\mathrm{Cm}$ and $C 2 / \mathrm{m}$ for the initial- $\beta^{\prime \prime}$ and pre- $\beta^{\prime \prime}$ phases, respectively). The atomic positions are still based on the parent $\mathrm{Al}$ host matrix (space group $F m \overline{3} m$ ), and thus these structures are fcc superstructures. During annealing at a temperature of $453 \mathrm{~K}$ for about $30 \mathrm{~min}$ (this is the industrial bake hardening treatment) the nanoclusters transform from pre- $\beta^{\prime \prime}$ to the $\beta^{\prime \prime}$ phase, a transformation associated with a large increase in hardness. Eventually the $\beta^{\prime \prime} \mathrm{Mg}_{5} \mathrm{Si}_{6}$ phase is formed which is the optimum hardening precipitate., ${ }^{4,17}$ The transformation is achieved through collective shifts of individual atomic columns, resulting in a product phase that is no longer an fcc superstructure. The starting point of the transformation (fcc-type phases ${ }^{6,17,19}$ ) and the end point (non-fcctype $\beta^{\prime \prime}$ phase $e^{4,5,20,21}$ ) are known both experimentally and theoretically from the literature. The columnar shift follows directly from a comparison of the atomic positions before and after the transformation. In this work, we calculate the 


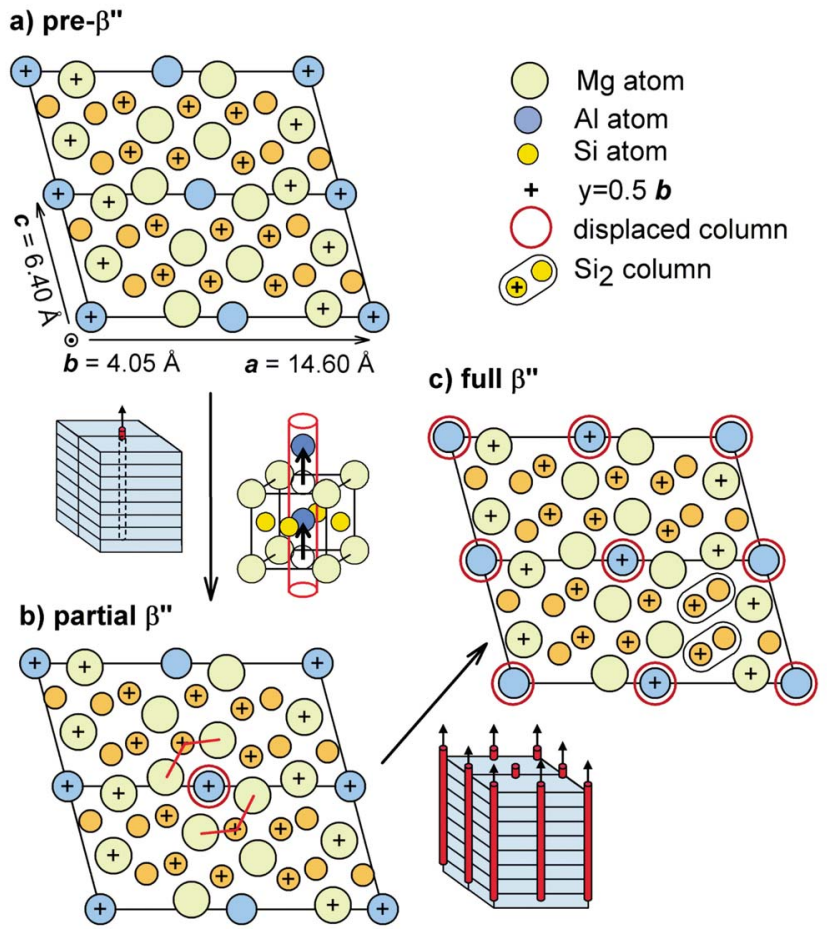

FIG. 3. (Color online) Phase transformation from pre- $\beta^{\prime \prime}$ to $\beta^{\prime \prime}$ at composition $\mathrm{Mg}_{4} \mathrm{Si}_{6} \mathrm{Al}$. The shift of the $\mathrm{Al}$ atoms from $y=0.0$ to $y$ $=0.5$ actually corresponds to shifts of atomic columns because the structure is periodic along the monoclinic $b$ axis (the viewing direction). The lattice dimensions are fully commensurate with the $\mathrm{Al}$ lattice; see Fig. 4(a). (a) Pre- $\beta^{\prime \prime}$ configuration, fcc type. (b) Partial $\beta^{\prime \prime}$ configuration after the shift of the central atomic column. (c) Full $\beta^{\prime \prime}$ configuration when all columns have shifted. A few $\mathrm{Si}_{2}$ columns, characteristic of the needle-type structures, are indicated.

activation energy for the shift shown schematically in Fig. 1. The phase transformation is shown in Fig. 3 for the composition $\mathrm{Mg}_{4} \mathrm{Si}_{6} \mathrm{Al}$. Figures 3(a) and 3(c) illustrate the cell dimensions and atomic coordinates of the pre- $\beta^{\prime \prime} \mathrm{Mg}_{4} \mathrm{Si}_{6} \mathrm{Al}$ structure and the $\beta^{\prime \prime} \mathrm{Mg}_{4} \mathrm{Si}_{6} \mathrm{Al}$ structure, respectively. A comparison with pure $\mathrm{Al}$, as displayed on the right-hand side of Fig. 4(a), shows that the pre- $\beta^{\prime \prime}$ structure is an fcc-based structure. Figures $4(\mathrm{~b})-4(\mathrm{~h})$ show the pre- $\beta^{\prime \prime}$ structure for various compositions. Considering Fig. 3, the transformation from pre- $\beta^{\prime \prime}$ to $\beta^{\prime \prime}$ is achieved by shifting the atoms at relative positions $(0, y, 0)$ and $\left(\frac{1}{2}, y, 0\right)$, the $\mathrm{Al}$ atoms in Fig. 3, along the $b$ axis with a magnitude of $\Delta y=0.5$ (a displacement of $0.202 \mathrm{~nm}$, half the $\mathrm{Al} \mathrm{fcc} \mathrm{lattice} \mathrm{parameter).}$

\section{COMPUTATIONAL DETAILS}

Despite the century-long investigations discussed above, the atomistic mechanism leading to the formation of the $\beta^{\prime \prime}$ phase is still insufficiently understood. In order to calculate the energy difference $\Delta U$ between the pre- $\beta^{\prime \prime}$ and $\beta^{\prime \prime}$ phases and the activation energy $E_{\text {act }}$ of the transition, we have calculated the energy change as function of the shift (Fig. 2). The structures are taken to be fully commensurate with the Al lattice displayed in Fig. 4(a). The energies of all structures

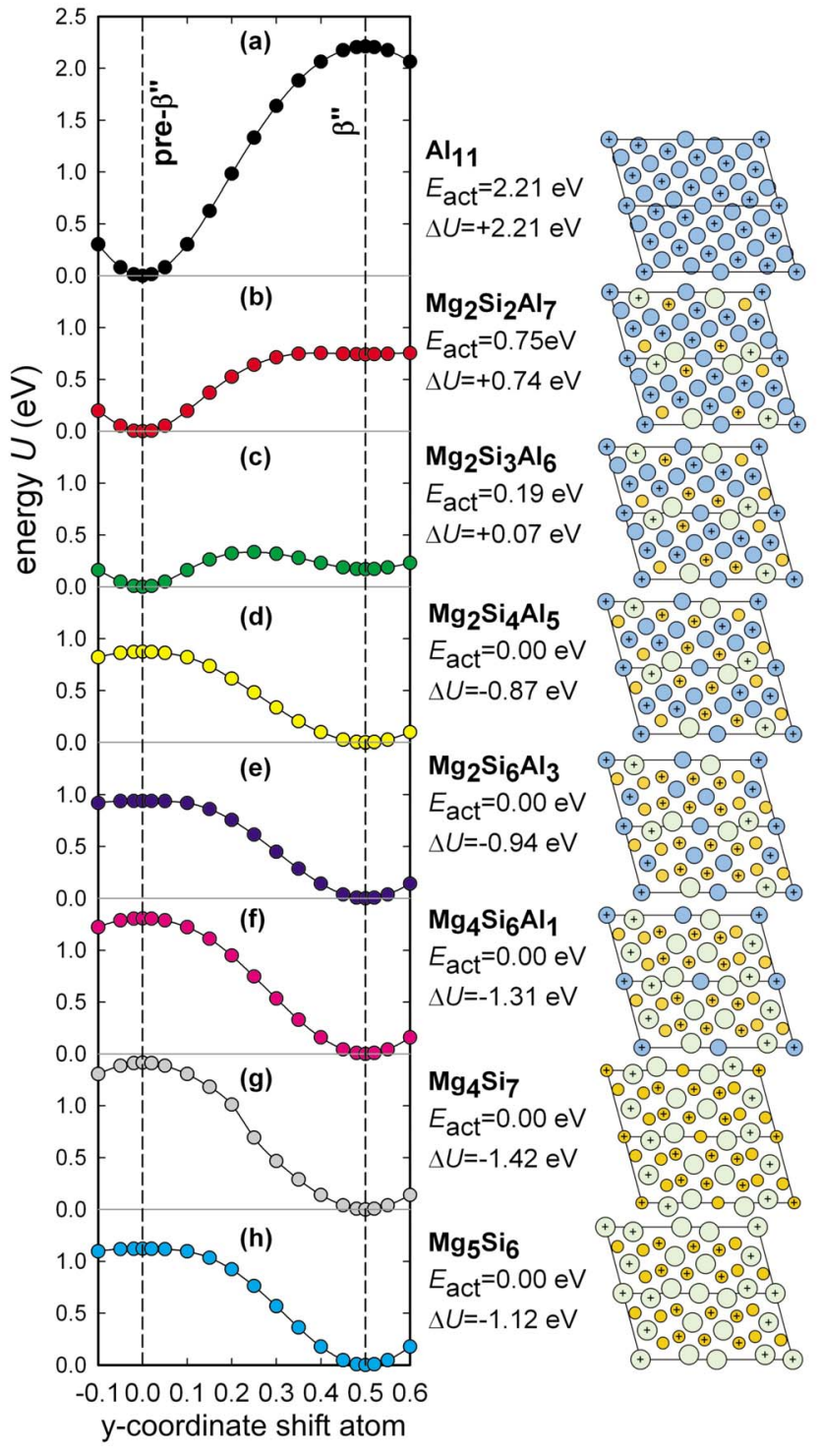

FIG. 4. (Color online) Left-hand side: energy change per supercell as a function of the displacement $y$ of the shift atom (i.e., energy change per atom in the shift column). See Fig. 2 for definitions of the energies $E_{\text {act }}$ and $\Delta U$. The shift constitutes the phase transition form pre- $\beta^{\prime \prime}$ to partial $\beta^{\prime \prime}$ and is schematically displayed in Figs. 3(a) and 3(b) Right-hand side: supercell structures of the pre- $\beta^{\prime \prime}$ phases with composition varying from pure Al to $\mathrm{Mg}_{4} \mathrm{Si}_{7}$ and $\mathrm{Mg}_{5} \mathrm{Si}_{6}$. Note that for the latter two, the central shift atoms are $\mathrm{Si}$ and $\mathrm{Mg}$ atoms, respectively.

were calculated using the ultrasoft (US) pseudopotential VASP code, ${ }^{22-24}$ employing the generalized gradient approximation (GGA) ${ }^{25}$ Energy cutoff and $k$-point convergence was ascertained to give a precision in the energy of $0.5 \mathrm{meV} /$ atom. All calculations were performed with an energy cutoff for the wave functions of $200 \mathrm{eV}$. The density of the Monkhorst-Pack $k$-point grid was increased until the above-mentioned precision was reached. A $k$-point grid of $6 \times 20 \times 8$ (pertaining to the reciprocals of the $a, b$, and double- $c$ axes, respectively) was required for the supercells of monoclinic structures displayed in Figs. 3 and 4. Atomic 
relaxation of the structures was stopped when consecutive ionic iterations differed less than $0.03 \mathrm{meV} / \mathrm{atom}$. In order to ensure that the energies correspond to the same minimumenergy path (MEP), the nudged elastic band (NEB) method was used as implemented in the VASP code, ${ }^{26}$ using a spring force constant of $-5.0 \mathrm{eV} / \AA^{2}$. Crystallographic details of the pre- $\beta^{\prime \prime}$ phases $\mathrm{Mg}_{4} \mathrm{Si}_{6} \mathrm{Al}, \mathrm{Mg}_{4} \mathrm{Si}_{7}$, and $\mathrm{Mg}_{5} \mathrm{Si}_{6}$ displayed in Fig. 4 can be found elsewhere. ${ }^{19,20}$

\section{RESULTS AND DISCUSSION}

As explained above, the transformation from the pre- $\beta^{\prime \prime}$ to the $\beta^{\prime \prime}$ phase consists of a shift of an atom along $0.5 b$ in the unit cell. Because of the periodicity, this one-atom shift actually represents the shift of a column of atoms extending along the $b$ axis (Figs. 1 and 3). Assuming that there is an energy barrier that has to be crossed for the atomic column to shift by $0.5 b$, it is unlikely that all columns will shift simultaneously. Therefore, only one atomic column, the one at the center of Fig 3(a), is shifted by $0.5 b$, giving the structure displayed in Fig. 3(b). Eventually, the edge columns will shift also, giving the material a full $\beta^{\prime \prime}$ configuration shown in Fig. 3(c). The atomic positions displayed in Figs. 3 and 4 correspond to the structure with the lowest energy found after relaxation of the atomic positions. The $0.5 b$ shift leads to a different configuration of the atoms surrounding the shift atom. In Fig. 3(b), it is clear that the angles between the $\mathrm{Mg}-\mathrm{Si}-\mathrm{Mg}$ atoms around the shift atom change considerably (the angles are indicated with red lines).

The transition energies required for the phase transformation from pre- $\beta^{\prime \prime}$ to $\beta^{\prime \prime}$ [Figs. 2, 3(a), and 3(b)] were calculated for a range of compositions with varying $\mathrm{Al}$ content (from pure $\mathrm{Al}$ to $\mathrm{Mg}_{5} \mathrm{Si}_{6}$ ). The energy of the structures is calculated as a function of the shift position and is displayed on the left-hand side of Fig. 4. The changes in energy are per supercell [consisting of two monoclinic cells displayed in Figs. 3(a) and 3(b) and at the right-hand side of Fig. 4] at constant volume. Because the supercell contains only one atom of the shift column, the energy changes are per atom in the shift column. The numerical values for the activation energy $E_{\text {act }}$ and the difference in energy between the two phases, $\Delta U$, are displayed also. For comparison, we have calculated the columnar shift in pure Al, shown in Fig. 4(a). There is a potential well at $y=0.0$ and the shift is obviously unfavorable with $\Delta U=+2.2 \mathrm{eV}$ per shift atom. In Fig. 4(f), at the composition $\mathrm{Mg}_{4} \mathrm{Si}_{6} \mathrm{Al}$, the position $y=0.0$ (pre- $\beta^{\prime \prime}$ configuration) does not correspond to a potential well, but to a saddle point. There is a continuous decrease in energy up to $y=0.5$ ( $\beta^{\prime \prime}$ configuration), where a potential well is positioned with a depth of $1.3 \mathrm{eV}$. Thus, the smallest fluctuation will cause a pre- $\beta^{\prime \prime}$ phase $(y=0.0)$ to transform to the $\beta^{\prime \prime}$ phase $(y=0.5)$. Therefore, the pre- $\beta^{\prime \prime}$ phases $\mathrm{Mg}_{2} \mathrm{Si}_{4} \mathrm{Al}_{5}$, $\mathrm{Mg}_{2} \mathrm{Si}_{6} \mathrm{Al}_{3}, \mathrm{Mg}_{4} \mathrm{Si}_{6} \mathrm{Al}, \mathrm{Mg}_{5} \mathrm{Si}_{6}$, and $\mathrm{Mg}_{4} \mathrm{Si}_{7}$ [Figs. 4(d)-4(h)] are all structurally unstable. The composition $\mathrm{Mg}_{2} \mathrm{Si}_{3} \mathrm{Al}_{6}$ [Fig. 4(c)] is the last stable pre- $\beta^{\prime \prime}$ phase, with $(\Delta U=$ $+0.07 \mathrm{eV})$ and the $E_{\text {act }}=0.19 \mathrm{eV}$. When the solute concentration increases only slightly to $\mathrm{Mg}_{2} \mathrm{Si}_{4} \mathrm{Al}_{5}$ [Fig. 4(d)], there is a dramatic change in the shape of the energy curve: the activation energy is zero and the energy gain is substantial
$(\Delta U=-0.87 \mathrm{eV})$. This energy profile corresponds to Fig. 2(c) and is characteristic of a displacive transformation.

A very small change in composition leads to a complete change of the characteristics of the transformation and in the stability of the pre- $\beta^{\prime \prime}$ phase. This transition is so fast that there is no time for a nucleation and growth process. Considering Fig. 2, subfigure (b) is characteristic of a nucleation and growth process, while subfigure (c) is characteristic of a displacive transformation (negligible activation energy). In Fig. 4, the profile of a nucleation and growth process does not occur: there is a direct transition from a unfavorable transformation [Figs. 4(c) and 2(a)] to a displacive transformation without activation energy and with a large energy gain [Figs. 4(d) and 2(c)]. The fact that the energy profile of a nucleation and growth process is absent in the series is evidence that the transformation is displacive in nature. The energy gain of the transformation is large, at least $0.9 \mathrm{eV}$ per atom in the shift column. This means that in a column $5 \mathrm{~nm}$ long, the displacement of the 12 atoms in that column yield an energy of $11 \mathrm{eV}$. Still, a change in composition is required for the transformation to occur, which brings about the possibility of a coupled displacive-diffusional transformation $^{27,28}$ (also called Widmanstätten type). In that case a displacive transformation proceeds as soon as solutes have segregated sufficiently (the growth of a transformed $\beta^{\prime \prime}$ needle at its end points is probably controlled by this process). Unfortunately, the mechanism of a displacivediffusional transformation cannot be studied by means of the current density functional theory calculations because that would require excessive computational resources: simulation of such complex behavior requires dynamic calculations in simulation cells with very large numbers of atoms.

A collective shift of a column of atoms is counterintuitive from the conventional point of view because it is assumed that there is always an energy barrier $\Delta E$ required for an atom to move [Figs. 2(a) and 2(b)] and that the collective shift of $n$ atoms then requires $n \Delta E$, which would in general render the energy for such a transformation too large to occur. However, in this case, we have shown that beyond the critical composition, $\Delta E=0$ and therefore $n \Delta E=0$ [Fig. 2(c)], so that there is no energy barrier for a columnar collective shift. The sizes of the $\mathrm{Mg}$ and $\mathrm{Si}$ atoms relative to the size of Al play an important role. Considering the nearestneighbor atoms around the central shift atom-e.g., in Figs. 3(a) and 3(b) - it is clear that the large Mg atoms "squeeze" the shifting $\mathrm{Al}$ atoms out of the plane $y=0.0$ towards the half-height plane $y=0.5$, where the small $\mathrm{Si}$ atoms move sideways to make room.

The question now arises why solute-rich pre- $\beta^{\prime \prime}$ structures are reported experimentally in the literature ${ }^{17}$ when they are structurally unstable according to the theoretical calculations. Deviations in the lattice parameters cannot stabilize the pre$\beta^{\prime \prime}$ structures: when the experimental lattice parameters ${ }^{4,17}$ are used in the calculations the activation energy for the shift remains zero. A more plausible explanation is that the experimental determination of the $y$ coordinate of the shift atom is incorrect. In the analysis of high-resolution transmission electron microscopy (HRTEM) images ${ }^{17}$ along the $b$ axis (which does not contain any information on the $y$ coordinates) it was concluded from image contrast (lateral $x, z$ 
shifts of the black dots) that all atoms were still on fcc lattice sites. This pre- $\beta^{\prime \prime}$ model also considered that interatomic distances would become unrealistic in a $\beta^{\prime \prime}$ arrangement. ${ }^{17}$ It was already reported previously that this consideration is invalid, which was found by quantum mechanical refinement of atomic positions. ${ }^{19}$ Our calculations show that the transformation into $\beta^{\prime \prime}$ can be favorable already at compositions with an $\mathrm{Al}$ content of 50 at. \%, which corresponds to early precipitates having dimensions as small as $5-10 \mathrm{~nm}$. This is much earlier than assumed up until now. It follows that pre$\beta^{\prime \prime}$ models for the more mature compositions $\mathrm{Mg}_{4} \mathrm{Si}_{6} \mathrm{Al}$, $\mathrm{Mg}_{2} \mathrm{Si}_{6} \mathrm{Al}_{3}$, and $\mathrm{Mg}_{4} \mathrm{Si}_{7}$ (Refs. 17 and 19-21) are not valid.

Considering Fig. 4, the precipitation process in $\mathrm{Al}-\mathrm{Mg}-\mathrm{Si}$ alloys follows a path whereby continuous compositional changes induce collective shifts resulting in crystal structures that cannot be considered as superstructures of the matrix. Although the compositional change is also coupled to an ordering transformation as has been found in previous theoretical work, ${ }^{12,15}$ it cannot be separated from the simultaneously occurring displacements. Already in very early stages the relaxation is so strong that the original parent lattice is obfuscated. This means that theoretical approaches such as cluster expansions, ${ }^{12-15}$ which consider superstructures only, cannot describe the actual precipitation process. Another consequence of this finding is that for precipitating systems where the precipitate structure is not a matrix superstructure it might nevertheless be possible to identify lattice correspondence through a path involving a combination of enrichment, ordering, and displacements. Obviously, this description reaches beyond the ideas of classical nucleation theory where a nucleus already has all the properties of the new phase.

The incoherency of the $\beta^{\prime \prime}$ structure with the $\mathrm{Al}$ fcc lattice at the nanocluster-matrix interface is also of importance. Considering Fig. 5, the top of the shifting column will be pushed into the Al lattice, where there is no space. Therefore, a vacancy at the top of the column is required for the columnar shift. Alternatively, when a vacancy is present somewhere along the shift column, both ends of the column will shift in opposite directions towards the vacancy position in the middle. The presence of the vacancy is not a severe requirement as vacancies are commonly present in the initial supersaturated solid solution. Actually, the maturing of the precipitate by means of the order-disorder transformation (replacement of $\mathrm{Al}$ atoms with $\mathrm{Mg}$ and $\mathrm{Si}$ atoms in an ordered configuration) depends entirely on vacancy-assisted migration; i.e., vacancies are required for the solute diffusion. The diffusion energies of $\mathrm{Mg}$ and $\mathrm{Si}$ in $\mathrm{Al}$ are 1.25 and $1.22 \mathrm{eV}$, respectively, and include the formation enthalpy of Al vacancies, $0.69 \mathrm{eV}$ (Ref. 29). However, the vacancy can also be generated by the shift column itself. As shown above, the energy gain is so large that enough energy $(\sim 11 \mathrm{eV}$ for a 5-nm-long column) is available for the formation of a a)

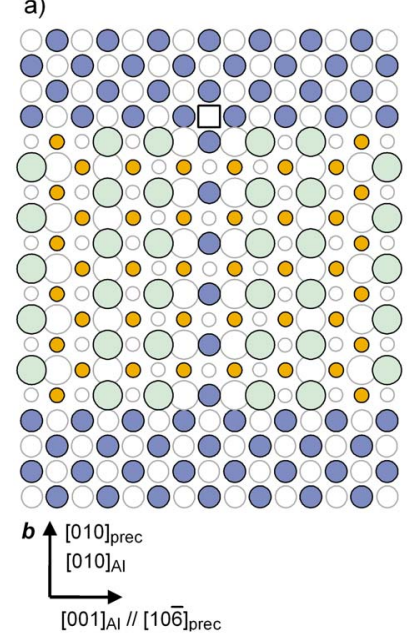

b)

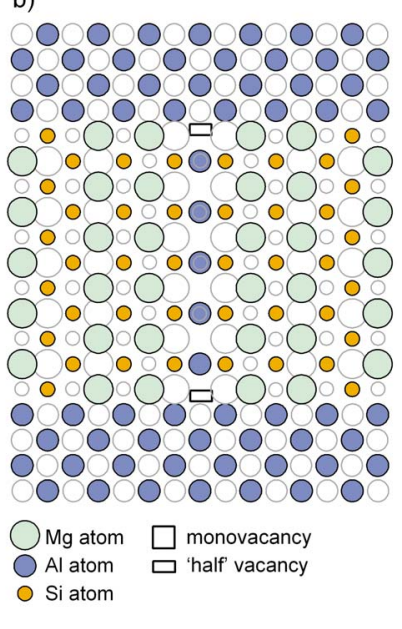

FIG. 5. (Color online) Illustration of the $0.5 b$ shift (upwards in the figure) of the column of $\mathrm{Al}$ atoms in the $\mathrm{Mg}_{4} \mathrm{Si}_{6} \mathrm{Al}$ precipitate embedded in fcc Al. The solid circles are atoms in the top $(100)_{\mathrm{Al}}$ plane $(x=0.5)$; the open circles are atoms in the bottom $(100)_{\mathrm{Al}}$ plane $(x=0.0)$. See Figs. 1 and 3 for the orientation relationships of the nanoclusters with the $\mathrm{Al}$ matrix. (a) Pre- $\beta^{\prime \prime}$ arrangement, before the $0.5 b$ shift and with an $\mathrm{Al}$ vacancy on top of the shift column. (b) $\beta^{\prime \prime}$ arrangement after the $0.5 b$ shift. Two "half" vacancies remain at the two ends of the shifted column.

vacancy or even the knock-out of an aluminum atom (formation of a Frenkel defect-i.e., a vacancy-interstitial pair).

\section{CONCLUSION}

In summary, we show that the precipitation process can only be described by a combination of inseparable transformations: enrichment of solute atoms, an order-disorder transformation within the nanoclusters and a columnar displacive transformation that does not require any activation energy. The simultaneous occurrence of a displacive transformation, which disrupts atomic coordinations and neighbor shells, makes it difficult to apply current state-of-the-art cluster expansion methods to the substitutional processes. Therefore, highly detailed first-principles investigations are required in these alloys. This mechanism for the evolution of precipitation in $\mathrm{Al}-\mathrm{Mg}-\mathrm{Si}$ alloys may explain also precipitation phenomena in other systems where the parent and precipitate phases appear unrelated.

\section{ACKNOWLEDGMENTS}

This research was funded by the Netherlands Institute for Metals Research (NIMR, Project No. 02EMM023), the Dutch Foundation for Fundamental Research on Matter (FOM), and the Dutch Foundation for Technology and Science (STW, Project No. DTF.7532). 
*Corresponding author. FAX: $\quad+31 \quad 152782351$. m.a.vanhuis@tudelft.nl

${ }^{1}$ A. Wilm, Metallurgie (Halle) 8, 225 (1911).

${ }^{2}$ A. Guinier, Nature (London) 142, 569 (1938).

${ }^{3}$ G. D. Preston, Philos. Mag. 26, 855 (1938).

${ }^{4}$ H. W. Zandbergen, S. J. Andersen, and J. Jansen, Science 277, 1221 (1997).

${ }^{5}$ S. J. Andersen, H. W. Zandbergen, J. Jansen, C. Træholt, U. Tundal, and O. Reiso, Acta Mater. 46, 3283 (1998).

${ }^{6}$ J. H. Chen, E. Costan, M. A. van Huis, Q. Xu, and H. W. Zandbergen, Science 312, 416 (2006).

${ }^{7}$ J. W. Christian, The Theory of Transformations in Metals and Alloys, 3rd ed. (Pergamon Press, Oxford, 2002), Chaps. 4, 10, and 16.

${ }^{8}$ J. W. Cahn and J. E. Hilliard, J. Chem. Phys. 31, 688 (1959).

${ }^{9}$ J. W. Cahn, J. Chem. Phys. 42, 93 (1965).

${ }^{10}$ M. Volmer and A. Weber, Z. Phys. Chem., Stoechiom. Verwandtschaftsl. 119, 277 (1926).

${ }^{11}$ R. Becker and W. Döring, Ann. Phys. 24, 719 (1935).

${ }^{12}$ J. Wang, C. Wolverton, S. Müller, Z.-K. Liu, and L.-Q. Chen, Acta Mater. 53, 2759 (2005).

${ }^{13}$ G. L. W. Hart, V. Blum, M. J. Walorski, and A. Zunger, Nat.
Mater. 4, 391 (2005).

${ }^{14}$ A. Van de Walle, Nat. Mater. 4, 362 (2005).

${ }^{15}$ C. Wolverton, Modell. Simul. Mater. Sci. Eng. 8, 323 (2000).

${ }^{16}$ M. Murayama and K. Hono, Acta Mater. 47, 1537 (1999).

${ }^{17}$ C. D. Marioara, S. J. Andersen, J. Jansen, and H. W. Zandbergen, Acta Mater. 49, 321 (2001).

${ }^{18}$ T. Ecipier, Adv. Eng. Mater. 8, 1197 (2006).

${ }^{19}$ C. Ravi and C. Wolverton, Acta Mater. 52, 4213 (2004).

${ }^{20}$ M. A. van Huis, J. H. Chen, H. W. Zandbergen, and M. H. F. Sluiter, Acta Mater. 54, 2945 (2006).

${ }^{21}$ M. A. van Huis, J. H. Chen, M. H. F. Sluiter, and H. W. Zandbergen, Acta Mater. 55, 2183 (2007).

${ }^{22}$ G. Kresse and J. Furthmüller, Phys. Rev. B 54, 11169 (1996).

${ }^{23}$ G. Kresse and J. Hafner, J. Phys.: Condens. Matter 6, 8245 (1994).

${ }^{24}$ G. Kresse and J. Furthmüller, Comput. Mater. Sci. 6, 15 (1996).

${ }^{25}$ J. P. Perdew and Y. Wang, Phys. Rev. B 45, 13244 (1992).

${ }^{26}$ G. Henkelman and H. Jónsson, J. Chem. Phys. 113, 9978 (2000).

${ }^{27}$ G. B. Olson, H. K. D. H. Bhadeshia, and M. Cohen, Acta Metall. 37, 381 (1989).

${ }^{28}$ J. M. Howe, Mater. Sci. Eng., A 438-440, 35 (2006).

${ }^{29}$ Y. Du et al., Mater. Sci. Eng., A 363, 140 (2003). 